\title{
Assessing Deaf Cultural Competency of Physicians and Medical Students
}

\author{
Lisa Hoang • Sheila F. LaHousse • Melanie C. Nakaji • \\ Georgia Robins Sadler
}

Published online: 23 July 2010

(C) The Author(s) 2010. This article is published with open access at Springerlink.com

\begin{abstract}
The Medical Students, Cancer Control, and the Deaf Community Training program (DCT) intended to create physicians who were culturally competent to care for deaf patients were evaluated. DCT medical students $(n=$ $22)$, UCSD medical faculty $(n=131)$, and non-DCT medical students $(n=211)$ were anonymously surveyed about their perceptions related to deaf patients, deaf cultural competency, and interpreter use. The faculty and non-DCT medical students displayed less knowledge than the DCT students. These findings suggest that training medical students in deaf cultural competency can significantly increase their capacity to care for community members and reduce the health disparities experienced by this community.
\end{abstract}

Keywords Deaf cultural competency · Physicians · Medical students

\section{Introduction}

Of the 8.8 million North Americans who are deaf [1], nearly a million belong to the Deaf community [2]. The Deaf community is distinguished by its preference for using American Sign Language (ASL) and its distinct culture.

L. Hoang · S. F. LaHousse • M. C. Nakaji · G. R. Sadler

Moores UCSD Cancer Center,

3855 Health Sciences Drive \#0850,

La Jolla, CA 92093-0850, USA

G. R. Sadler $(\square)$

UCSD School of Medicine,

3855 Health Sciences Drive \#0850,

La Jolla, CA 92093-0850, USA

e-mail: gsadler@ucsd.edu
There are many studies showing that members of the Deaf community experience health disparities due to language barriers, which contribute to lower health literacy $[1,3]$. The problem is exacerbated by the fact that most physicians are not adequately prepared to provide linguistically and culturally competent care for deaf patients [4]. Many lack knowledge about deaf culture and deaf patients' rights [5-7]. Healthcare providers report discomfort with deaf patients, limited understanding of deaf culture, and a belief that deaf patients do not trust them [8-10].

Many physicians do not realize how few words can be lip-read $(30 \%)$ [2, 8, 10, 11]. Failure to communicate effectively can lead to mistakes and this contributes to deaf patients' preference to avoid physicians $[8,10,12]$ and an increasing number of lawsuits [1]. While many physicians believe interpreters should be used when interacting with a deaf patient, few actually schedule them $[2,11,13]$, while others are unaware of their legal obligation to provide interpreters [6]. Compounding the problem, many deaf people themselves do not understand their rights to interpreting services [2].

Further contributing to the distrust of physicians has been the medical community's view of deafness solely as a pathophysiological disease that needs to be "cured," rather than a unique culture and language that warrants respect $[2$, 14]. Other deaf patients perceive that physicians act in a paternalistic manner that effectively reduces their autonomy $[1,2]$ and associate their healthcare experiences with stress and strong negative emotions $[2,15]$.

Since many healthcare barriers stem from health care providers' lack of community-specific cultural and linguistic competency when working with members of underserved groups, training providers to be culturally and linguistically competent should help reduce those barriers [16-18]. For example, when physicians can sign, deaf patients report that 
they were more likely to follow recommended health maintenance behaviors, visit their physicians regularly [5], and feel greater satisfaction with the clinical experience [2].

Given the lack of training in deaf culture and the limited number of providers who sign, the University of California, San Diego (UCSD) School of Medicine created the NCI-funded fellowship program, Medical Students, Cancer Control, and the Deaf Community Training program (DCT). The fellowship was created to train a small cohort of its medical students in ASL and deaf culture within the context of a cancer control curriculum. The goal was to have them ultimately become the medical partners of Deaf community leaders who were advocating for improved access to health information and care.

\section{Methods}

Hypothesis

The DCT medical students will demonstrate greater knowledge of deaf culture and deaf patients than UCSD's non-DCT medical students and faculty.

\section{Intervention}

As the first part of this 2-year training program, fellows participated in a deaf culture training program. They were asked to master a curriculum of self-paced reading materials that would provide a sound understanding of deaf culture. Over the first 2 years of school, they completed six quarters of ASL classes and one summer at Gallaudet University's residential ASL/deaf culture immersion program. Throughout this time, the previously mastered cultural concepts were reinforced. While in school, students practiced their ASL and cultural competency by interacting with the community and by providing the Deaf community with workshops about health promotion. Students complete their mandatory research projects on a topic related to the Deaf community and take fourth year rotations where they interact with the Deaf community [19]. Fellows received an $\$ 8,000$ year stipend as compensation for the extra burden the program's placed on the fellows during their medical studies and as a mode of retention.

\section{Study Design}

DCT students, non-DCT students, and faculty were invited to take part in an institutional review board (IRB)approved, anonymous survey with six multiple choice and 28 true-false questions plus an "I don't know" option. To maintain participant anonymity, IRB had approved an implied consenting process, which placed the consent statement at the top of the survey page and considered return of the survey as sufficient evidence of implied consent. To keep the survey brief, the only sociodemographic data gathered was whether they were faculty, DCT, or non-DCT.

The survey questions were created based on a review of the literature [2, 5-11, 13], the project team members' prior knowledge, and guidance from the project's Deaf community advisory group. Questions were related to: (1) commonly held misperceptions of deafness and deaf culture, (2) common difficulties experienced by deaf patients in the clinical settings, (3) errors commonly made when providers work interpreters in the clinical setting, and (4) the participants' prior exposure to the Deaf community. The survey also asked respondents to list up to five problems they thought deaf patients may face when hospitalized (Table 1). The survey was extensively pilot tested prior to dissemination.

\section{Recruitment of Participants}

From April 2007 to May 2008, 780 medical school faculty and 640 non-DCT students and 25 DCT students from the graduating classes of 2007-2011 were sent the survey via list-serve and Survey Monkey [20], making anonymous responses possible. Although participants were reached in various ways, they were instructed to complete and return only one survey. While it was not possible to separate the medical school faculty with MD degrees $(N=525)$ from those with other terminal degrees, the survey instruction declared that the survey was directed only to faculty with direct patient care responsibilities.

\section{Data Analysis}

All data were entered and analyzed using SPSS version 14.0 [21]. Responses were summed to create an overall, continuous sum score, where greater scores indicated more knowledge. A binary coding system $(1=$ correct, $0=$ incorrect) was used for all items. The Knowledge sum score was analyzed using analysis of variance and $t$ tests $[22,23]$. Chi-square tests were used to compare responses to individual items among the three study groups. A significant difference is described as a $p$ value greater than 0.05 .

\section{Description of the Sample}

Of the 372 surveys returned, nine were omitted because respondents reported that they were non-clinical faculty, not medical students, or did not self-identify. Participation was $25 \%(n=130 / 525)$ for faculty, $33 \%(n=211 / 640)$ for non- 
Table 1 DCT medical students, faculty and non-DCT medical students group comparisons on knowledge multiple choice items

\begin{tabular}{lll}
\hline $\begin{array}{l}\text { Assessing knowledge of deaf cultural competency } \\
\text { in a medical setting }\end{array}$ & $\begin{array}{l}\text { DCT medical } \\
\text { students }\end{array}$ & $\begin{array}{l}\text { School of } \\
\text { medicine faculty }\end{array}$ \\
& Percent correct\% $(n)$ & $\begin{array}{l}\text { Non-DCT } \\
\text { medical } \\
\text { students }\end{array}$ \\
\hline
\end{tabular}

Item 1: a cochlear implant

A. Will allow a deaf adult to immediately begin hearing and understanding oral conversations (incorrect)

B. Destroys any residual hearing in the ear that the patient may have had (correct)

C. Corrects for any type of hearing loss (incorrect)

D. Is desired by at least $90 \%$ of deaf people (incorrect)

E. Do not know (incorrect)

Item 2: in a medical setting, it is the right of the deaf patient

A. To express a preference for a particular interpreter (correct)

B. To be provided with an interpreter by the practitioner (correct)

C. To determine how much personal information he/she wants to disclose in an interpreted situation (correct)

D. Do not know (incorrect)

Item 3: the hospital has arranged for you to give a presentation on an important health topic with the assistance of an ASL interpreter.

The audience, which consists mainly of deaf patients, are all socializing prior to the presentation. You are ready to begin your presentation. You should:
A. Stand on stage and wait patiently for the audience to settle down (correct)
B. Flick the lights on and off several times in order to get the audience's attention (correct)
C. Clap loudly (incorrect)
D. Ask the interpreter to sign that you are ready to begin (correct)
$4.5(1)$
$95.5(21)^{* *}, * * * *$
$-$
$22.7(5)^{* *, * * * *}$
E. Do not know (incorrect)
A. Place the interpreter beside the patient. The patient and the interpreter are facing the provider (incorrect)
B. Place the interpreter beside the provider. The provider and the interpreter are facing the patient (correct)
C. Place the interpreter at an equal distance between the provider and the patient (incorrect)
D. Do not know (incorrect)
Item 5: You have a deaf couple who refuse to have their newborn baby's hearing tested. You should:
A. Tell them this is required by law, and that it has to be done for their baby's benefit (incorrect)
B. Tell them it is their decision, but explain that this lack of knowledge will put their baby at risk (incorrect)
C. Accept their decision (correct)
D. Do not know (incorrect)
$56.8(113)$
$-$
$42.1(85)$
$7.4(15)$
$-$

Item 6: you are in the Emergency Department (ED) and you call for a patient several times. Others in the ED point to a person reading a magazine and say "She's deaf." You should

A. Approach the patient and gently tap her on the shoulder (correct)

B. Approach the patient and call their name louder (incorrect)

C. Approach the patient, making small gestures in her field of vision to try to get her attention (correct)

D. Do not know (incorrect)

Question (correct answer)

1. Only $30 \%$ of the English language can be accurately lip read (true)

2. You are running considerably behind schedule. Your deaf patient is waiting with his/her interpreter. The interpreter is ethically bound to wait with the patient until you are ready to see them (false)

$\begin{array}{lll}81.8(18)^{* *}, * * * * & 46.7(57) & 51(103) \\ - & - & - \\ 22.7(5)^{* *} & 53.3(65)^{* * * * * *} & 28.2(57) \\ - & - & - \\ & & \\ \text { Percent correct\% (n) } & & 13.5(26) \\ 86.4(19)^{* *, * * * *} & 17.1(20) & 18.7(36) \\ 31.8(7) & 16.2(19) & \end{array}$


Table 1 (continued)

Assessing knowledge of deaf cultural competency

in a medical setting
DCT medical

students

Percent correct $\%(n)$
School of

medicine faculty
Non-DCT

medical

students
3. ASL is a pictorial language that produces a word-for-word translation of what is being said in English (false)

4. The majority of hearing parents with deaf children never learn to sign (true)

5. When communicating with a deaf patient through an interpreter, you should face the interpreter and explain to the interpreter what the patient needs to know (false)

6. Trying to help cure your patient's deafness should be your top priority (false)

7. Because deaf people rely upon printed forms of information, their literacy is equal to or better than the general public (false)

8. A good interpreter will be able to step out of his/her interpreting role in order to explain to the provider what the patient is really trying to say (false)

9. When there is a dominant source of light, such as a window, your deaf patient should be seated with his/her back to the light source and you should be seated facing the light source (true)

10. For an infant, there is very little that can be done to improve an infant's hearing due to its age (false)

11. When speaking to a deaf patient through an interpreter you should speak each word very slowly, to allow the interpreter time to sign or fingerspell your words (false)

12. For most members of the deaf community, English is their primary language (false)

13. When a deaf patient is hospitalized, the entire staff should be notified that the patient is deaf (true)

14. When hiring an interpreter, the minimum time per session is two hours (true)

15. At the end of the health care visit, the interpreter should again review the information with the patient (false)

16. Early in the conversation, your patient mentions to you that he has Usher's syndrome. This information will influence how you communicate with him (true)

17. Deaf patients generally do not participate in support groups such as those that help patients cope with disease or death. The main reason for this is due to the language barrier (true)

18. On average, deaf patients report that they are unable to convey adequate information to their doctors (true)

19. Less than $50 \%$ of physicians who have deaf patients use a certified interpreter (true)

20. Working with other minority and/or disabled population will adequately prepare a physician to work with the deaf (false)

21 . Ninety percent of deaf people have hearing parents (true)

22. If a child is found to have a hearing loss, you should also refer the child to an optometrist (true)

23. It is the patients' responsibility to schedule the interpreter if they think one will be needed (false)

24. You have complicated surgical information to communicate to a deaf patient, so it would be wise to tell the patient to bring along a friend or family member to assist with the interpretation (false)

25. If the patient requests an interpreter for a visit with their health care provider, it is the patients' responsibility to pay for the interpreter (false)

26. If a deaf patient requests an interpreter, you may ask your nurse, who has taken several semesters of ASL classes, to interpret for the consultation (false)

27. If you suspect hearing loss in an infant, you should make a note to recheck the infant's hearing on the next visit (false)

\begin{tabular}{|c|c|c|}
\hline $100.0(22)^{* *, * * * *}$ & $69.2(81)$ & $71.0(137)$ \\
\hline $90.9(20)^{* *, * * * *}$ & $13.7(16)$ & 9.9 (19) \\
\hline $90.9(20)$ & $75.0(87)$ & $80.8(156)$ \\
\hline $100.0(22)^{*}$ & $83.8(98)$ & $88.0(169)$ \\
\hline $95.5(21)^{* *}, * * * *$ & $34.5(40)^{* * * * *}$ & $23.3(45)$ \\
\hline $72.7(16)^{*, * * * *}$ & $46.6(54)^{* * * * *}$ & $34.2(66)$ \\
\hline $77.3(17)^{* * * *}$ & $56.0(65)^{* * * * * *}$ & $38.3(74)$ \\
\hline $72.7(16)$ & $69.2(81)^{* * * * * *}$ & $54.4(105)$ \\
\hline $86.4(19)^{* *}, * * * *$ & $55.6(65)^{* * * * * *}$ & $39.9(77)$ \\
\hline $90.9(20)^{* *}, * * * *$ & $38.3(44)$ & $29.0(56)$ \\
\hline $77.3(17)$ & $74.1(86)^{* * * * * *}$ & $57.8(111)$ \\
\hline $27.3(6)^{* *, * * * *}$ & $5.1(6)$ & $2.6(5)$ \\
\hline $31.8(7)^{* *, * * * *}$ & $6(7)$ & $6.3(12)$ \\
\hline $45.5(10)^{* * * *}$ & $33.3(39)^{* * * * * *}$ & $14.2(27)$ \\
\hline $68.2(15)^{* *, * * * *}$ & $18.8(22)$ & $18.0(34)$ \\
\hline $81.8(18)^{* *, * * *}$ & $49.6(58)$ & $56.8(108)$ \\
\hline $81.8(18)^{* *}, * * * *$ & $41.9(49)$ & $40.0(76)$ \\
\hline $95.5(21)^{* * * *}$ & $82.9(97)^{* * * * * *}$ & $65.3(124)$ \\
\hline $77.3(17)^{* *, * * * *}$ & $44.4(52)$ & $36.8(70)$ \\
\hline $63.6(14)^{* * * *}$ & $43.5(50)^{* * * * *}$ & $30.0(57)$ \\
\hline $81.8(18)^{* *}, * * * *$ & $39.7(46)$ & $41.9(80)$ \\
\hline $95.5(21)^{* *}, * * * *$ & $44.4(52)$ & $38.9(74)$ \\
\hline $90.9(20)^{* *}, * * * *$ & $40.2(47)$ & $45.3(86)$ \\
\hline $95.5(21)^{* *}, * * * *$ & $45.3(53)$ & $39.5(75)$ \\
\hline $13.6(3)$ & $22.2(26)^{* * * * *}$ & $12.8(24)$ \\
\hline
\end{tabular}

$100.0(22)^{* * * * * * *}$

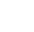

90.9


Table 1 (continued)

\begin{tabular}{|c|c|c|c|}
\hline $\begin{array}{l}\text { Assessing knowledge of deaf cultural competency } \\
\text { in a medical setting }\end{array}$ & $\begin{array}{l}\text { DCT medical } \\
\text { students } \\
\text { Percent correct } \%(n)\end{array}$ & $\begin{array}{l}\text { School of } \\
\text { medicine faculty }\end{array}$ & $\begin{array}{l}\text { Non-DCT } \\
\text { medical } \\
\text { students }\end{array}$ \\
\hline \multirow{2}{*}{$\begin{array}{l}\text { 28. American Disabilities Act requires an interpreter be } \\
\text { present whether the patient wants one or not (false) }\end{array}$} & $68.2(15)^{* *, * * * *}$ & $23.1(27)$ & $19.1(36)$ \\
\hline & $\mathrm{M}(\mathrm{SD})$ & $\mathrm{M}(\mathrm{SD})$ & $\mathrm{M}(\mathrm{SD})$ \\
\hline Total summary score $(\mathrm{TF}+\mathrm{MC})^{\mathrm{a}}$ & $\begin{array}{l}26.90(5.34) \\
n=21\end{array}$ & $\begin{array}{l}17.07(5.81)^{* * * * * * *} \\
n=105\end{array}$ & $\begin{array}{c}13.79(6.39) \\
n=179\end{array}$ \\
\hline
\end{tabular}

${ }^{a}$ Total score was created by summing all possible correct answers across the 34 items. This score ranges from 0 to 39 , greater scores indicate more knowledge

${ }^{*} p \leq 0.05$, chi-square difference between DCT medical students and faculty are significant

$* * p \leq 0.01$, chi-square difference between DCT medical students and faculty are significant

$* * * p \leq 0.05$, chi-square difference between DCT medical students and non-DCT medical students are significant

$* * * * p \leq 0.01$, chi-square difference between DCT medical students and non-DCT medical students are significant

$* * * * * p \leq 0.05$, chi-square difference between faculty and non-DCT medical students are significant

$* * * * * * p \leq 0.01$, chi-square difference between faculty and non-DCT medical students are significant

DCT medical students, and $88 \%(n=22 / 25)$ for DCT students.

\section{Results}

The hypothesis was supported. The DCT students had a significantly higher overall knowledge score $(M=26.9)$ than the faculty $(M=17.07)$ and the non-DCT students $(M=$ 13.79; $p \leq 0.01$; Table 1). For example, DCT students were significantly more likely to know that most deaf children are born to hearing parents, deaf patients seldom use support groups, and the different modes of communications and difficulties associated with each mode. Table 1 shows the cumulative scores as well as the scores for each of the three groups broken down by individual questions.

We anticipated that for the faculty, DCT, and non-DCT students, having prior exposure to the Deaf community would predict higher cultural knowledge scores. The linear regression analysis showed that this prediction held true only for the faculty $(B=3.839, \mathrm{SE}=1.751, p<0.05)$. However, faculty with prior experience still scored far below the DCT students, but significantly higher $(M=$ 17.51) than non-DCT students with prior exposure $(M=$ 13.92; Table 2).

Further analyses were conducted to compare the total knowledge scores between faculty and non-DCT students based on whether they reported having a deaf or hard-ofhearing person in their immediate social circle. For those who reported not having a deaf or hard-of-hearing person in their social circle, faculty had significantly higher average knowledge scores $(M=17.73)$ than the non-DCT students $(M=13.66)$. There was no difference in knowledge scores between faculty and non-DCT students who reported having a deaf or hard-of-hearing person in their social circle (Table 2).

Among those who reported awareness of deaf culture, the faculty $(M=18.09)$ had greater knowledge scores than the non-DCT students $(M=14.23)$. All faculty (who did, $M=$ 17.82; and did not, $M=13.44$ report wanting to take an ASL class in the past) had higher knowledge scores compared to non-DCT students in the corresponding categories $(M=$ $16.70, M=14.28$, respectively). Faculty who reported never taking an ASL class had significantly higher knowledge scores $(M=17.01)$ than non-DCT students in the same category (Table 2).

Participants were asked to list up to five problems they felt a deaf person would have when hospitalized, other than the obvious difficulties of communicating with their healthcare provider or the inability to use the room's telephone. Responses to this open-ended question were qualitatively analyzed. Those responses that were too ambiguous or not specific to difficulties experienced by deaf patients were omitted from analysis. Response were clustered by the primary author and two independent researchers $(85 \%$ inter-rater agreement) [24, 25], culminating into 15 themes. For those responses in which there was a disagreement, a consensus among the three researchers was reached. Results from this qualitative thematic analysis [25] are outlined in Table 3. 
Table 2 Comparison of faculty and non-DCT medical student

Percentage that responded "yes" to questions regarding past exposure to deaf and hard-of-hearing persons

Faculty\% (n) Non-DCT student $\%(n)$

$\begin{array}{ll}87.5(112)^{* * *} & 44.2(92) \\ 46.1(59)^{* * *} & 14.9(31) \\ 82.8(106) & 84.1(175) \\ 4.7(6) & 2.9(6) \\ 31.3(40)^{* * *} & 58.2(121) \\ \text { Faculty M (SD) } & \text { Non-DCT student M (SD) }\end{array}$

Have you ever had exposure to deaf or hard-of-hearing people?

Has there ever been a deaf or hard-of-hearing person in your social circle?

Are you aware that there is a deaf culture?

Have you ever taken an American Sign Language (ASL) class?

Have you ever wanted to take an ASL class?

Comparison of knowledge scores based on exposure of participant to deaf or hard-of-hearing persons

Has prior exposure to deaf or hard-of-hearing persons?

Yes Average total score

No Average total score

Has deaf or hard-of-hearing person in social circle?

Yes Average total score

No Average total score

Are you aware that there is a deaf culture?

Yes Average total score

No Average total score

$15.51(5.40)^{*}, * * * n=93$

$13.92(6.01) n=78$

$13.67(7.83) n=12$

$13.68(6.70) \mathrm{n}=101$

$16.34(5.45) n=50$

$14.54(5.57) n=26$

$17.73(6.10)^{* * *} n=55$

$13.66(6.53) n=153$

$18.09(4.95)^{* * *} n=88$

$14.23(6.27) n=152$

$11.76(7.13) n=17$

$11.30(6.65) n=27$

Have you ever taken an American Sign Language (ASL) class?

Yes Average total score

No Average total score

$18.20(4.39) n=5$

$15.67(8.08) n=3$

$17.01(5.89)^{* * *} n=100$

$13.76(6.39) n=176$

Ever wanted to take an ASL class in the past?

Yes Average total score

$17.82(6.06)^{* * *} n=34$

$13.44(6.33) n=105$

No

Average total score
$16.70(5.70)^{* *} n=71$

${ }^{*} p \leq 0.05$, within group mean difference between faculty with without exposure was significant

$* * p \leq 0.05$, mean difference between faculty and non-DCT medical students are significant

${ }^{\wedge}$ Mean difference between faculty and non-DCT medical students are significant $(\mathrm{p} \leq 0.01)$

The frequency with which the three groups endorsed each theme is reported in Table 3. Each group endorsed the 15 difficulties in unique ways. DCT students listed "understanding terms and medical language" as the number one difficulty experienced by deaf patients when hospitalized, while faculty and non-DCT students listed "communication with persons other than healthcare providers, (i.e., janitor or dietician)." The DCT students also were more likely to list maltreatment or mistreatment as a difficulty.

\section{Discussion}

Literature has shown that the Deaf community experiences barriers to the acquisition of health information and care $[1,2,4,5,8,9,11,14,16,26-28]$, which may be improved by increasing clinicians' cultural competency $[8-10,12,14,15,17,19,29,30]$. The DCT program was designed to address this issue. The data presented in this paper demonstrate that the DCT students scored signifi- cantly higher than faculty and non-DCT students on knowledge related to cultural competency within the Deaf community.

Overall, the faculty performed significantly better than non-DCT medical students, especially on questions related to interactions with deaf patients (e.g., how to approach a deaf patient or what to do with children who have hearing loss; Tables 1 and 2). This observation may be the culmination of faculty's clinical work with deaf or hard-of-hearing persons. Exposure proves to be a differentiating factor even amongst the faculty because faculty without exposure scored the same as non-DCT students in general. However, having a deaf or hard-of-hearing person in one's social circle did not translate into a better understanding of their difficulties in healthcare. These results suggest that exposure to members of the Deaf community in a clinical setting may be most beneficial in increasing physicians' cultural competency when a program such as the DCT is not available. Medical schools can benefit from this finding by offering classes or self-paced learning modules that can create deaf cultural competency of medical students, faculty, and attendings. 
Table 3 Cumulative ranking of most frequently listed perceived problems that hospitalized deaf patients may encounter in a hospital setting

1. Communication with personnel other than provider (e.g., dietician, janitor, etc.)

2. Emergency or warnings

3. Emotions (fear/confusion)

4. Understanding terms and medical knowledge

5. Knowledge of rights and interpreter services

6. Awareness of activities in hospitals heard in announcements

7. Maltreatment or mistreatment by medical staff

8. Privacy issues

9. Circumstances that inhibit communication (e.g., vision impairments or immobility of hands)

10. Difficulty with the radio or television

11. Limited medical knowledge/lack of health literacy

12. Contacting family and friends

13. Social support

14. Distrust of providers

15. Decreased awareness of surroundings (e.g. People in the room, coming up from behind)

Top three reported problems by group

DCT-medical students
1.Understanding medical language $(19 \%)$

2.Communication $(17 \%)$

3.Maltreatment or mistreatment by medical staff $(17 \%)$

\author{
Medical school faculty \\ Non-DCT medical students \\ 1.Communication $(33 \%)$ \\ 1.Communication $(22 \%)$ \\ 2.Emergency or warnings $(19 \%)$ \\ 2.Emergency or warnings $(24 \%)$ \\ 3.Emotions such as fear or confusion (15\%) 3.Emotions such as fear or confusion $(12 \%)$
}

By evaluating the open-ended responses from the DCT and non-DCT students, it became clear that the DCT students were focusing on problems that extended beyond the Deaf community's physiologic differences (i.e., inability to hear). The issues listed by the DCT students including difficulty with understanding medical terms, as well as fearing or experiencing mistreatment or maltreatment is a point that would be considered only if one is knowledgeable of deaf culture and deaf history (Table 4).

Recruitment efforts were made to maximize sample sizes, but the number of DCT students was limited in comparison to the other study arms. The DCT student group was self-selected and given a funded fellowship as compensation for the program's extra burden, and thus does not necessarily represent the interest of all medical students. Thus, study results should be generalized with caution to other medical schools. There was also the potential for variation in the interpretation of a few survey questions that may have limited the interpretation of the results. For example, while it can be inferred that the faculty's exposure to deaf and hard-of-hearing people is equated with advancing years of clinical experience, the term "exposure" as used in the questionnaire did not specify the qualities of the exposure. Future studies may help to clarify this point. Future studies should also examine whether receiving only the deaf culture training educational materials without the more expansive training program (ASL coursework, ASL immersion, and ongoing ASL and deaf culture training throughout medical school), could produce the same desired level of cultural competency.

\section{Conclusion}

Deaf people face challenges in accessing health care which may be ameliorated by providing healthcare providers with cultural competency training. Understanding that the Deaf community is a linguistic and socio-cultural minority will help clinicians more effectively respond to issues of human diversity in the healthcare setting.

Acknowledgments The authors acknowledge the following sources of support for this study: NIH 5P30CA023100; NIH U56CA92079/ U56CA92081 and U54CA132379/U54CA132384; NIH/NCMHD 5P60MD000220; NIH 5R25CA65745; NIH R25CA101317; NIH R25CA108731; the California Endowment; Alliance Healthcare Foundation 99-99; The Komen for the Cure Foundation, San Diego Affiliate Grant Award; and UCSD Academic Senate Grant.

This project was created by an educational partnership consisting of: the Moores UCSD Cancer Center, Deaf Community Services of San Diego Inc., Bovee Productions, the National Association for the Deaf, and Gallaudet University. The authors wish to also thank the following additional organizations that made the participant recruitment process possible: Center on Deafness Inland Empire (CODIE) in Riverside, CA; Orange County Deaf Equal Access Foundation (OC DEAF) in Cypress (Orange County, CA); Deaf program with Disability Support Programs and Services at San Diego Mesa College in San Diego, CA; and the National Center on Deafness at California State University Northridge in Northridge, CA. 
Portions of the data included in this paper were presented as a poster at the October 2009 American Association for Cancer Education's Annual Conference, where it won the award for First Place.

Open Access This article is distributed under the terms of the Creative Commons Attribution Noncommercial License which permits any noncommercial use, distribution, and reproduction in any medium, provided the original author(s) and source are credited.

\section{References}

1. Harmer L (1999) Health care delivery and deaf people: practice, problems, and recommendations for change. J Deaf Stud Deaf Educ 4(2):73-110

2. Steinberg AG et al (2006) Health care system accessibility. Experiences and perceptions of deaf people. J Gen Intern Med 21(3):260-266

3. Chew LD, Bradley KA, Boyko EJ (2004) Brief questions to identify patients with inadequate health literacy. Fam Med 36(8):588-594

4. Barnett S (2002) Cross-cultural communication with patients who use American Sign Language. Fam Med 34(5):376-382

5. Barnett S, Franks P (2002) Health care utilization and adults who are deaf: relationship with age at onset of deafness. Health Serv Res 37(1):105-120

6. Ralston E, Zazove P, Gorenflo DW (1996) Physicians' attitudes and beliefs about deaf patients. J Am Board Fam Pract 9(3):167-173

7. Steinberg AG, Sullivan VJ, Loew RC (1998) Cultural and linguistic barriers to mental health service access: the deaf consumer's perspective. Am J Psychiatry 155(7):982-984

8. Iezzioni L et al (2004) Communicating about health care: observation from persons who are deaf or hard of hearing. Ann Intern Med 140(5):356-362

9. Iezzoni LI et al (2002) Satisfaction with quality and access to health care among people with disabling conditions. Int J Qual Health Care 14(5):369-381

10. Witte TN, Kuzel AJ (2000) Elderly deaf patients' health care experiences. J Am Board Fam Pract 13(1):17-22

11. Ebert DA, Heckerling PS (1995) Communication with deaf patients. Knowledge, beliefs, and practices of physicians. JAMA 273(3):227-229

12. Tamaskar $P$ et al (2000) Preventive attitudes and beliefs of deaf and hard-of-hearing individuals. Arch Fam Med 9(6):518-525, discussion 526
13. Shabaik $\mathrm{S}$ et al. (2009) A randomized educational trial on colorectal cancer in a community sample of deaf adults. J Cancer Educ (in press)

14. Philips BA (1996) Bringing culture to the forefront: Formulating diagnostic impressions of deaf and hard-of-hearing people at times of medical crisis. Prof Psy Res Prac 27(2):137-144

15. Sadler GR et al (2001) Bringing health care information to the deaf community. J Cancer Educ 16(2):105-108

16. Steinberg AG et al (2002) Deaf women: experiences and perceptions of healthcare system access. J Womens Health (Larchmt) 11(8):729-741

17. Barnett S (1999) Clinical and cultural issues in caring for deaf people. Fam Med 31(1):17-22

18. Lock E (2003) A workshop for medical students on deafness and hearing impairments. Acad Med 78(12):1229-1234

19. Farber JH, Nakaji MC, Sadler GR (2004) Medical students, deaf patients and cancer. Med Educ 38(11):1201

20. Richards J et al (1999) Deaf strong hospital: an exercise in crosscultural communication for first year medical students. $\mathrm{J} \mathrm{U}$ Rochester Med Center 10(5):5-7

21. SPSS for Windows Version 14.0.0. (2005) SPSS Inc.: Chicago

22. Cohen $J$ et al (2003) Applied multiple regression/correlation analysis for the behavioral sciences, 3rd edn. Lawrence Erlbaum Associates, Hillsdale, NJ

23. Kleinbaum DG et al (2007) Applied regression analysis and multivariable methods, 4th edn. Duxbury Press, Pacific Grove, CA

24. Miles MB, Huberman AM (1994) Qualitative data analysis: an expanded source book. Sage, Thousand Oaks

25. Patton MQ (2002) Qualitative evaluation and research methods, 3rd edn. Sage, Newbury Park

26. Barnett S (2002) Communication with deaf and hard-of-hearing people: a guide for medical education. Acad Med 77(7):694-700

27. Collins J (1996) Medical stress - deaf women as patients. Deaf Worlds 12(1):8-11

28. Ekstrom I (1994) Communicating with the deaf patient. Plast Surg Nurs 14(1): 31-32, 35

29. Sadler GR et al (2001) Bringing breast cancer education to deaf women. J Cancer Educ 16(4):225-228

30. Smith MC, Hasnip JH (1991) The lessons of deafness: deafness awareness and communication skills training with medical students. Med Educ 25(4):319-321

The Deaf Community is denoted by a capital " $D$ " not be confused with the audiological term "deaf." 